\title{
Should Hospitalization be an Outcome for Vaccine Trials? \\ Godofreda Vergeire-Dalmacion*
}

Department of Pharmacology and Toxicology and Department of Clinical Epidemiology, University of the Philippines Manila, Philippines

"Corresponding author: GodofredaVergeire-Dalmacion, Department of Pharmacology and Toxicology and Department of Clinical Epidemiology, University of the Philippines Manila, Philippines, E-mail: jody.dalmacion@gmail.com

Received date: April 02, 2018; Accepted date: August 08, 2018; Published date: August 15, 2018

Copyright: $\odot 2018$ Vergeire-Dalmacion G. This is an open-access article distributed under the terms of the Creative Commons Attribution License, which permits unrestricted use, distribution and reproduction in any medium; provided the original author and source are credited.

\begin{abstract}
After the mass immunization of Filipino school children with Dengvaxia $₫$, there has been a continuing report of deaths, unofficially 41 among the vaccines. The country is endemic for Dengue and experiences epidemic proportion of the disease annually. The overall efficacy rate of Dengvaxia can be misleading because there are no cross protection across all sero types of dengue and the vaccine efficacy varies widely across the 4 dengue serotypes. The early efficacy study showed 4 deaths in the vaccine group but none in the placebo. One of the deaths was a case of acute disseminated encephalomyelitis, which was adjudged temporally related to Dengvaxia ${ }^{\circledR}$. The Antibody Dependent Enhancement phenomenon for dengue that gives a more serious second episode of the virus might not only be increasing the probability of hospitalization but also increasing the case fatality at least for Filipino children. The Number Needed to Vaccinate (NNV) of 46 in the 28th day after vaccination was favourable for the vaccine. Disturbingly, the NNV when calculated in about the 3rd year after immunization became negative and now interpretable instead as Number Needed to Harm. Vector control, early detection and timely and appropriate management of dengue remain the safer option for dengue control in the Philippines.
\end{abstract}

Keywords: Dengue; Dengue vaccine; NNV; NNH; Immunisation; Hospitalisation

The uncertainties on the efficacy and safety of Dengvaxia continue to haunt doctors, parents, and lawmakers alike in the Philippines. After the mass immunization of about 830,000 children with Dengvaxia, there has been a continuing report of deaths, unofficially 41 to date among those who received the vaccine from 2016-2017.

Countries in the Asia Pacific region, including the Philippines, were one of the sites for the Dengvaxia Clinical Trial. In 2014, the Efficacy Trial of Dengvaxia covering the period after completion of the 3 doses up to the 28th day was published by Capeding et al. [1]. An overall efficacy rate of $56.5 \%$ was reported but it can be misleading since the efficacy rates for each serotype varied widely. The secondary analyses showed low (35\%) and inconclusive efficacy against serotype 2, which is considered as a weak point of Dengvaxia and is still under research [2]. The Number Needed to Vaccinate (NNV) based on 117 Dengue cases out of 6848 in the Vaccine group for all serotypes and 133 Dengue cases in 3424 under the Placebo group is 46 . It means 46 individuals have to be vaccinated in order to avert 1 case of Dengue [1]. However, NNV for Dengue type 2 is 322.5 which are almost double the numbers you have to vaccinate to prevent just 1 case of Dengue. In contrast, the NNV for the trivalent Influenza Vaccine for community-dwelling seniors is 40 [3], and 1031-3050 for childhood influenza with an efficacy of 50\% for ages 6-23 months [4]. Although NNV has been used to assess the cost effectiveness of several vaccines, a systematic review on NNV has shown no definite threshold for a favourable NNV [5].

Disturbing are the reports of Capeding et al. of 4 deaths as early as close to a month after the 3rd dose in the Vaccine Group giving the probability of 1 death out of 1712 versus 0 in the Placebo group. One of the deaths was due to acute disseminated encephalomyelitis which occurred 7 days after vaccine administration, indicating a strong temporal relationship with Dengvaxia administration. The rest of the deaths were due to injuries and were adjudged as not related to Dengvaxia. Methinks it is too soon to discount the possibility of a relationship between these deaths with the vaccine. Many years back cases of vehicular accidents and falls especially among the elderlies were reported after benzodiazepine use [6]. Had the relationship between Benzodiazepines and injurious fall been conveniently ignored based on the lack of "rational" basis, elderly patients on benzodiazepines will remain at risk from this disabling harm.

Unfortunately, the results of the Dengvaxia ${ }^{\oplus}$ Trial involving participants who reached 25 months post immunization cast more doubts and concerns on the long term safety and efficacy of the vaccine. The results of CDY 14 which is the Dengvaxia Trial in the Asia Pacific Region revealed an increased incidence of hospitalization and severe dengue in the vaccine group especially among those seronegative prior to the Dengvaxia administration [7].

Twenty seven cases of hospitalization for Dengue out of 6778 $(39.83 \%)$ were reported in the vaccine group and only $13 / 3887$ (33.44\%) for the placebo group. We calculated a NNV of (-) 6896 for the overall group, the negative value changing the NNV instead to the Number Needed to Harm (NNH), meaning that out of 6896 children vaccinated with Dengvaxia, 1 will be hospitalized. The negative NNV $(-502)$, persisted in the 3 rd year for all age groups and slowly decreased in the 4th year. Unlike NNT/NNV which is favourable if it carries a low value, a high $\mathrm{NNH}$ is more ideal.

Why use hospitalization to measure outcome? Paradoxically, vaccine trials target healthy individuals and are meant to prevent illnesses, not to treat an existing disease. If drug regulation has not changed, hospitalizations, like deaths, disability, and congenital anomalies, is considered severe adverse events and are warning signals about the safety of the product being studied. 
Since Dengvaxia ${ }^{\circledR}$ does not confer cross-protection for other strains of Dengue, Sanofi perhaps had expected from the onset the higher possibility of a second dengue episode in endemic settings. The second episode will likely be more severe and naturally fatal based on the proposed mechanism of Halstead known as Antibody Dependent Enhancement phenomenon first described after the Dengue epidemic in Thailand and the Philippines [8,9]. Clinically, the best Dengue vaccine is one that protects the child not only from a first infection but also attenuates or minimizes the severity of a second episode of Dengue which disappointingly Dengvaxia failed to demonstrate. Despite being endemic to the Philippines [9], there was very little protection given by Dengvaxia as a whole to children younger than 9 years during which time seropositivity should be higher than the other countries which re not endemic for the infection. The expected case fatality for Dengvaxia among severe and hospitalized cases is low at $0.44 \%$. Why then have we been getting reports of death allegedly as early as after a month from vaccination? Is there be a possibility that the case fatality rate had been increased with Dengvaxia which was not evident in sample size-constrained clinical trials but now visible in the real world with patients close to reality and when the numbers exposed is more than 830,000 ?

Was it worth spending 3.5 billion for the Dengue vaccine, a cost which is equivalent to the entire dengue control program of the country? Dengue has killed 25,000 worldwide which is only $2.5 \%$ of the 500,000 reported cases of severe Dengue (WHO Fact Sheet updated 2017). These statistics are not remarkable compared to the 500,000 deaths worldwide from Influenza. Moreover, only $25 \%$ of Dengue is symptomatic while $75 \%$ are subclinical or resolves spontaneously without catastrophic outcomes and relatively low case fatality rate.

Is the vaccine safe? Even the old vaccines such as MMR are known to be associated with some severe adverse reactions. For example, after decades of MMR use by 1.8 Million people and 3 million doses, the incidence of probable or indeterminate adverse events of MMR was calculated to be $5.3 / 100,000$. Only 1 death was recorded due to febrile seizure from MMR. In contrast, Dengvaxia with only 6851 exposed population already showed 4 deaths and higher risk for hospitalization by the third year post immunization [10].

The deaths and autopsy findings were described by the forensic expert in the Public Attorney's Office as rapid and characterized by massive bleeding as well as enlargement of almost all internal organs. The same expert recounted in a radio interview the gross abnormalities of the 4 fatalities to consist of "...internal bleeding, enlarged organs and petechial rashes and one death occurring within 24 hours after experiencing dengue like symptoms". Presently, there is no room for Dengvaxia in the Philippines or perhaps anywhere else where the course of Dengue infection is similar to the country.

Logically, deaths will be significantly averted if we can set up a program that can screen the etiology of fever in a child at the community level and sift out the Dengue cases for confirmation and be able to administer timely, appropriate and aggressive management. Children in the Philippines affected with dengue often succumb because of late detection ergo late or no interventions. The vector mosquito is abundant and all serotypes of dengue are present in the Philippines. With the growing number of deaths reported after the mass immunization, I strongly feel there is no advantage for the vaccine at this moment in time over a sustained and reliable vector control coupled with early detection of Dengue infections.

\section{References}

1. Capeding MR, Tran NH, Hadinegoro SR, Ismail HI, Chotpitayasunondh $\mathrm{T}$, et al. (2014) Clinical efficacy and safety of a novel tetravalent dengue vaccine in healthy children in Asia: A phase 3, randomised, observermasked, placebo-controlled trial. The Lancet 384: 1358-1365.

2. Sauer J (2018) Dengvaxia: The World's First Dengue Vaccine. The Prognosis 6.

3. Kolber M, Lau D, Eurich D, Korownyk C (2014) Effectiveness of the trivalent influenza vaccine. Can Fam Physician 60: 50.

4. Lewis EN, Griffith MR, Szilagyi G, Zhu Y, Edwards KM, et al. (2007) Childhood Influenza: Number needed to vaccinate to prevent 1 hospitalization or outpatient visit. Pediatrics 120: 467-472.

5. Hashima A, Danga V, Bolotina S, Crowcroft N (2014) How and why researchers use the number needed to vaccinate to inform decision making-A systematic review. Vaccine 33: 753-758.

6. Pariente A, Dartigues JF, Benichou J, Letenneur L, Moore N, et al. (2008) Benzodiazepines and injurious falls in community dwelling elders. Drugs Aging 25: 61-70.

7. Hadinegoro SR, Arredondo-garcía JL, Capeding MR, Deseda CT, Chotpitayasunondh R, et al. (2015) Efficacy and long-term safety of a dengue vaccine in regions of endemic disease. New Eng J Medi 373: 1195-1206.

8. Halstead SB (1988) Pathogenesis of dengue: Challenges to molecular biology. Science 239: 476-481.

9. Urrugga E, Edillo F, Erasmo JN, Alera MT, Yoon IK et. al (2017) Disease burden of dengue in the Philippines: Adjusting for underreporting by comparing active and passive dengue surveillance in Punta Princesa, Cebu City. Am J Trop Med Hyg 96: 887-898.

10. Patja A, Davidkin I, Kurki T, Kallio MJ, Valle M, et al. (2000) Serious adverse events after measles-mumps-rubella vaccination during a fourteen-year prospective follow-up. Pediatr Infect Dis J 19: 1127-1134. 